\title{
Towards combining the innovation ecosystem concept with intermediary approach to regional innovation development
}

\author{
Leyla Gamidullaeva ${ }^{1, *}$ \\ ${ }^{1}$ Penza State University, 440026, Krasnaya str., 40, Penza, Russia
}

\begin{abstract}
Research and development is becoming a global phenomenon implemented on a purely collaborative basis. This leads to the need to search for partners, resources and ideas outside the company. Active development of information and digital technologies in the international practice has promoted gradual emergence of new forms of innovation intermediaries, which have not yet been applied in Russia. We suppose these new forms could be successfully implemented into the Russian innovation ecosystem. The present research is devoted to searching ways to unite the innovation ecosystem concept with the theory of innovation intermediation, emphasizing specific types of innovation intermediation and fundamental mechanisms thereof, supporting incentives and the role in an innovation ecosystem. The research results may be implemented into managerial practices in order to improve and increase the innovation feature and competitiveness of regional innovation systems of the Russian Federation and of concrete enterprises in particular.
\end{abstract}

\section{Introduction}

The number of objects of the innovation infrastructure (business incubators, business accelerators, technological parks, innovation centers, centers for technology transfer and commercialization etc.) is expanding at a very rapid pace. Unfortunately, the same could not be said about their effectiveness. It should be noted that the problem with overcoming gaps between the state research and the development sector, the sector of universities' R\&D and the private sector of the economy still remains. An increase in the number of intermediaries in the innovation sphere, whose functions are performed by objects of the innovation infrastructure, does not solve the task of integration of the national innovation system of Russia. An unsystematic character of innovative infrastructure establishment and its institutional weakness determine poor interaction between science and industry. In recent years, the concept of innovation ecosystem is gaining strength and recognition. This theory describes evolving interrelations between economic actors, changes of innovation activity models and their relations with external environment (Mercan and Goktas, 2011). According to this approach, development, implementation and distribution of social innovations considerably influence innovation growth and economic development in the

* Corresponding author: gamidullaeva@gmail.com 
framework of a socio-economic system. From our point of view the integration of the ecosystem approach and the innovation intermediation theory in order to research regional innovation systems is of particular interest.

The present study is devoted to searching ways to unite the innovation ecosystem concept with the theory of innovation intermediation, emphasizing specific types of innovation intermediation and fundamental mechanisms thereof, supporting incentives and the role in the innovation ecosystem. The research results may be implemented into managerial practices in order to improve and increase the innovation feature and competitiveness of regional innovation systems of the Russian Federation and of concrete enterprises in particular.

\section{Theoretical Background}

Scholars as well as practitioners have been increasingly pointing out the significance of the innovation ecosystem concept. The inability of classical innovation models to form successful strategies that speed up innovation distribution has provoked the emergence of the theory of innovation ecosystem. Yawson (2009) argues that there is a need to extend the evidence-based platform for innovation policy and science. The basic postulate of the innovation ecosystem theory is a substantial increase of the actor's capabilities through collaboration with other actors (Adner, 2006). The ecosystem thinking has been elevated to the national level (Jackson 2011; Metcalfe \& Ramlogan 2008; Yawson 2009). National and regional systems of innovations (Lundvall 1992, Cooke et al. 1997) have considered the idea of innovations as open and interactive. However, these theories are incapable of identifying successful strategies that drive innovations at the national level (Yawson, 2009).

Mercan \& Göktaş (2011) indicate that the innovation ecosystem is composed of two parts -1 ) economic actors and economic relations; 2) technologies, institutions, cultural and sociological elements. Hence, the innovation ecosystem is presented as a set of different systems and networks.

Researches of innovation processes carried out in past decades highlight the crucial importance of networks in achieving successful innovations. It leads to a complete change of the traditional model that describes innovations as a linear sequence starting with fundamental research followed by product development, production and marketing. Currently, innovations are considered to be an interactive process that requires intensive moves, ideas, information, facts both inside and outside the company.

The term "innovation network" has a broad understanding. This term includes innovation networks promoted by major European Programmes that link firms and other establishments from different countries, sectors into the concrete innovation environment. The environment is used by SMEs to run different innovation projects.

If the purpose of collaboration is not related to the innovation activity, the fact of collaboration with other companies enhances innovation opportunities of the company. Put simply, the network can be considered as a group of cooperating companies, a particular group may represent an innovation system, a value chain, a cluster, or any other type of inter-firm relationships.

Networks are able to link up two firms only, or they may be extended. A greater emphasis may be placed on a single activity, for example, education, or on a specific technical problem, or on an activity in the value chain framework. Network's life span may be limited by the period of one project's life cycle or by the terms of all projects. The network can be backed by formal relations.

Obviously, the nature of networks is extremely diverse. Networks are so complex that researchers are unable to determine the "ideal" network. Although, the experience shows that some of those features can be particularly beneficial. The "ideal" network should have 
all key participants closely located, maintain long-term and stable relations and consider every link as a key part of the participants' business strategy. The participants should also develop close informal relations and constantly improve them; all network participants must have equal rights and opportunities. There must a sufficient number of participants to provide a rich source of knowledge and ensure manageability.

The network is considered to be a new method of organization of economic activity, which makes it possible to compensate disadvantages inherent in deeply integrated structures. Networks are able to strengthen advantages of specialization through easy access to external sources without increasing the interest rate. The network resource allocation method enables to perform transactions through a network of individuals and institutions engaged in mutually beneficial and supportive activities, rather than through fragmented exchanges and administrative decrees. In this sense, the ecosystem thinking comes close to what is called an open innovation (Fig. 1).

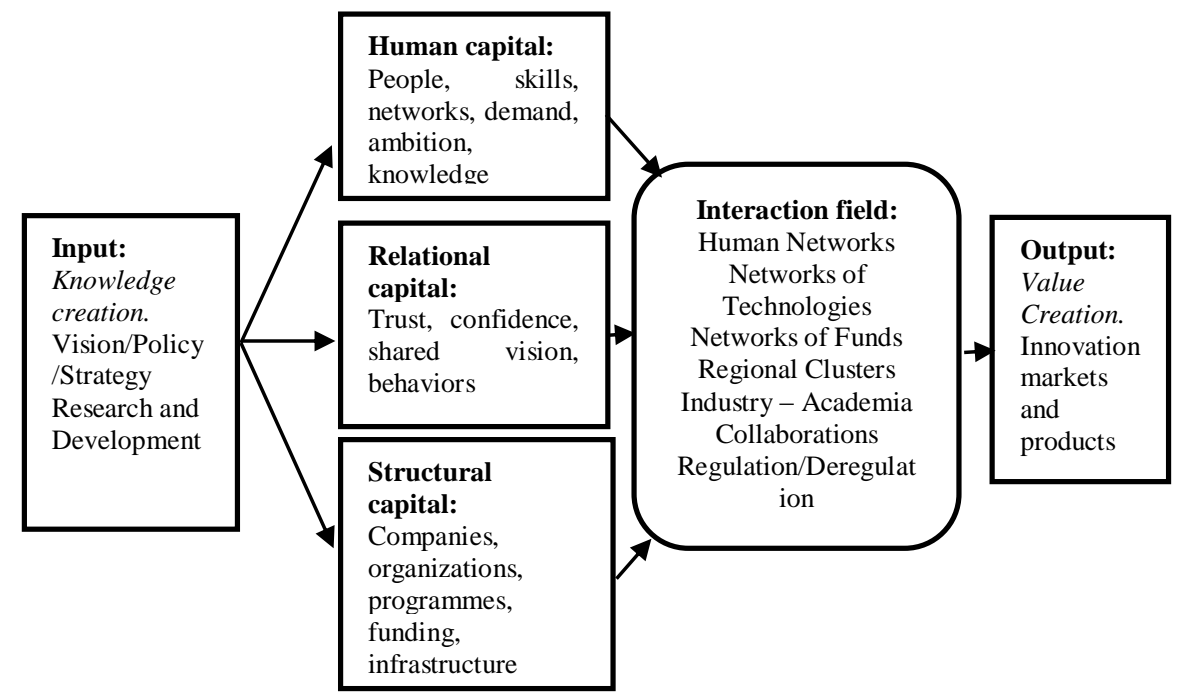

Fig. 1. Key Elements, Input and Output of Regional Innovation Ecosystem.

The gist of open innovation is that knowledge sharing is more beneficial than its accumulation within the company. The concept considers the research and development process as an open system. Therefore, sources of new knowledge may be inside or outside the company.

Herewith, on the other hand, due to high speed information exchange a competitive advantage in the local markets may be reached through fast implementation of new technologies created outside the company.

According to H. Chesbrough (2003) in the framework of the open innovation paradigm the flow of knowledge creates a knowledge landscape which is an open access space with a great deal of databases of scientific ideas (Chesbrough, 2003). The knowledge landscape emerges due to incoming (inflows) and outgoing (outflows) of knowledge. These ideas are accessible for the use and implementers (specialists) may be employed by other companies.

Therefore, the innovation ecosystem is considered to be an ecosystem based on collaboration, where innovations are created on the cooperative basis in the specific network environment based on horizontal integration of legally independent participants. Innovation developments on the basis of the network model of innovation system organization constitute the latest model of the innovation process. I. Ansoff and A. 
Campbell have greatly contributed to mutual understanding of collaboration importance (Ansoff, 2007). They have shown that synergetic effects of the innovation economy are unique implicit resources, which may be found when instead of independent closed activities the company chooses cooperative activities in the field of common experience utilization, common personnel and equipment usage. Business, therefore, joins other economic actors, diversifies its activities and functions, creates web sites, joint ventures, logistics systems, agro-industrial complexes, innovation clusters, strategic alliances, merges with and acquires other companies, searches for synergy effects in various forms of firms' associations, in holding company models, consortiums, in spatial distribution of productive forces.

Some empirical studies have found that firms' collaboration with external partners may provide their effectiveness and speed up the innovation process (Chesbrough, 2003). According to the open innovation approach, actors needed more knowledge flows to accelerate the innovation process and broaden markets. The adaptation of technologies from other spheres has proved particularly effective in firms' innovation activity development. To support these processes there are being developed and already exist many different types of innovation intermediaries.

The intermediaries have an effect when a firm can't use its ideas inside for own products and services, and, thus, transferring to the market is considered as the only possible way to commercialize innovations. Complicated global changes in the economic environment have caused serious challenges for firms forcing them to create value by working in alliances and networks. Some authors (van Lente et al, 2003) highlight that intermediaries' significance is in maintenance of creation and coordination of networks of interdependent and different actors. These agents are called to coordinate firms within networks through contracts, agreements, etc.

For many years innovation technology parks, business incubators and regional innovation centers have been considered as the most prevalent types of intermediaries. Rapid development of digital technologies has resulted in emergence of new types of innovation intermediation, helping firms obtain technological solutions in two-sided technology and idea markets. These include, for example, NineSigma, Innocentive, Yet2.comin, and others. Living Labs, in particular, are able to facilitate the process of diffusing innovation, new technologies, intellectual property and licensing in the innovation system (Benassi \& Minin, 2009).

Howells (2006) reveals about 10 main functions of the intermediaries in the field of innovation, which are still insufficiently studied. There is a need to analyze the opportunities and limitations of implementation of these functions in the context of Russian regional innovation ecosystems. In addition, there is a lack of scientific studies devoted to the search for ways to connect the innovation ecosystem theory and innovation intermediation based on digital technologies. There is also a growing need to point out all future prospects of using new trends in digital services and innovation entrepreneurship for the regional governance.

\section{Results}

The Russian practice of establishing the innovation infrastructure for Russian regional users' innovations - the destination of the lion's share of the budgetary resources - has been developed de facto guided by the logic of industrialization mainly based on replication of production and infrastructure projects. Guided by its technological and innovation modernization policy Russian authorities have created a great amount of regional innovation intermediaries such as business incubators, technological parks, business accelerators, innovation centers, etc. Despite these efforts, Russian regional users' 
innovations have demonstrated negative results for more than 10 years. Innovation process participants should actively collaborate and develop the experience of joint projects on creation and commercialization of innovations. It is considered to be extremely important to shape a cardinally new methodological approach to understanding of the principles and mechanisms of the innovation system.

Gloor's (2006) model of Collaborative Innovation Networks implies that innovations are increasingly becoming more interactive, and economic growth involves various groups of society forming and unfolding a certain innovation ecosystem.

I. Ansoff and A. Campbell (Campbell \& Luchs, 2004) have greatly contributed to understanding the significance of collaboration. They have shown that synergistic effects of the innovation economy are unique implicit resources. Many researchers have extensively studied the phenomena of innovation entrepreneurship (Howells, 2006; Jeppesen and Lakhani, 2010; Verona, 2006). Some researchers have placed an emphasis on exploring the mechanisms of functioning of intermediation, which facilitate the creation of innovations (Winch and Courtney, 2007; Steward and Hyysalo, 2008 ), whereas others have focused on studying new forms of intermediation in the innovation sphere (Living Labs), which presuppose a high level of users' involvement in the innovation process altogether with other participants of the innovation system operating in both one-sided and two-sided markets (Almirall and Wareham, 2011; Rochet and Tirole, 2006 ). However, it is still unclear how the widely recognized concept of innovation ecosystem relates to the theory of innovation intermediation and what are its prospects regarding the Russian governance.

At the same time, there is growing practical evidence that the introduction of the concept of the innovation ecosystem boosts the research and development process making it more financially beneficial and diverse. As a result, this concept is being integrated into corporate strategies of different firms. This, in turn, requires development of new business models and granting new business opportunities in the markets of new ideas and new technologies. Nowadays, even powerful multinational companies are seeking to combine the internal network of structural divisions involved in the process of generation, storage, use and absorption of knowledge with external networks, including companies, universities and institutions related to the internal divisions of the company. It gives companies an opportunity to strengthen their internal capacity of generation, absorption and use of knowledge (Golichenko, 2011: 634). The authors have invented the term "absorptive capacity" identifying company's capacity and ability to evaluate and recognize the need for new external knowledge, accumulation and usage of the latter for commercial purposes and the ability to make long-term predictions of scientific advancement (Cohen and Levinthal, 1990). Their definition provides a broad understanding of the term as the identification and recognition of new information (internal and external) and its assimilation, application and usage for commercial purposes. This capacity is based on background knowledge that includes skills and knowledge about advanced developments of scientific and technological society.

Thus, there emerged the necessity of innovation intermediaries that should develop and implement a permanent systemic basis using companies' absorptive capacity in order to utilize accumulated scientific and technological knowledge. These intermediaries are intended to function between the scientific and market objects and the market demand. The main feature of such intermediaries is their ability to overcome the gap between the depth of knowledge and the width of knowledge, to associate different knowledge fields.

\section{Solutions and Recommendations}


Following the systems-based approach the research employed historical analysis, benchmarking, comparative analysis and questionnaire surveys of managers and clients of Russian innovation intermediaries, as well as in-depth interviews.

Generally, the use of innovative intermediaries pursues the following major objectives: to promote their own ideas and projects; external expertise of ideas; experience and knowledge exchange; partners search, mobilization of resources needed for project implementation. Thus, the development of innovation intermediation in regions provides an opportunity for companies to reduce costs of innovation development, to adjust sustainable innovation processes that are distributed in space, link them into a single chain of production, distribution, exchange and consumption of innovation products in regions.

Currently, the authorities all over the world display a trend to develop regional economic systems into regional innovation ecosystems. This in turn creates new challengers to innovation intermediaries. Hence, there are more actors within innovation ecosystems whose activities can be considered a by-product of their primary function (Nilsson and Sia-Ljungström, 2013), or whose activities are not initially aimed at innovation, although have an indirect influence on the innovation development. There may be found a lot of corresponding examples: non-researching firms that are focused on development activities, individuals who introduce their vision and predict future developments, providers of solutions mandated by their clients, trade unions or clubs that ensure interests of their clients (van Lente et al. 2003), and also various types of advisors and critics. These participants' activities have an indirect impact on the innovation system. Consequently, we propose a broader definition of 'innovation intermediaries'. From our point of view, innovation intermediaries should be considered as key innovation actors within the regional innovation ecosystem that include implicit elements deliberately functioning within innovation systems by coordinating innovation actors that are not primarily aimed at innovation, but whose activities have some impact on the innovation system.

\section{Functions of Innovation intermediaries}

According to the opinion of most authors, the most significant functions performed by innovation intermediaries in the innovation systems include the following:

1. Knowledge creation and dissemination.

2. Setting directions of research and development.

3. Entrepreneurial experimentation.

4. Creation of new markets.

5. Creation of legitimate entrepreneurial business environment.

6. Mobilization of resources.

7. Development of positive externalities.

Innovation intermediaries operate between actors of the innovation system, create the necessary links, form and create opportunities for development of relations and cooperation. In other words, they establish and coordinate relationships between actors of the innovation system. Their main task, therefore, must be understood not as the generation and implementation of innovations, but as the creation of opportunities and favorable conditions for the development of innovations.

An analysis of a significant amount of available literature devoted to description and systematization of innovation intermediaries allows us to reveal basic functions to be performed by them:

- knowledge transfer - linking innovative solutions and economic actors searching for solutions, linking start-up companies and large companies, providing a neutral space for 
interaction between various parties to create innovations, delivering scientific knowledge to end users;

- organization of cooperation between science and industry - supporting and coordinating cooperation between economic actors in the innovation system; providing opportunities for partners search to implement joint projects, as well as for search of necessary resources;

- coordination of demand - providing the interaction between end users and enterprises;

- specification of supply and providing more detailed information about the needs and requirements of users of innovations;

- knowledge production and collaboration - uniting knowledge of all interested parties in the innovation market; creating internal knowledge; transferring specialized knowledge;

- mobilization of scientific research;

- knowledge commercialization - marketing support to companies, sales organization, assistance in searching for necessary resources;

- forecasting - establishing consistency between the ongoing research and the needs of the real economy; analyzing foreseeable technological trends and developments;

- protective function - consulting on intellectual property, management and control;

- testing and evaluation - testing, diagnostics, creating product prototypes, validation, testing, technology assessment;

- standardization and regulation - consulting on standards, regulation and self-regulation.

The emergence of new forms of innovation cooperation is the result of development of information technologies. Increased use of the Internet has predetermined the emergence of virtual innovation intermediaries (for instance, virtual business incubators functioning in various subjects of the Russian Federation). These virtual innovation intermediaries have attracted increased attention because of its ability to cross geographic distances and perform multiple activities. Further development of this type of innovation intermediaries has continued with the occurrence of bilateral platforms operating in the technology markets. It is extremely important to integrate different sources of knowledge by transforming specific needs into the scientific interpretation and consulting economic actors about such questions as how to capture the value, to bridge the widening gap between value creation and value capture and how to benefit from external and internal flows of knowledge. That is, such intermediaries are more than just Internet platforms linking large organizations with solution providers. Some types of the intermediaries in the market of innovations contribute to the spread of technology, intellectual property and licensing.

\section{The importance of open innovations}

The significance of open innovations in a rapidly globalized world has been widely acknowledged in many studies. Research on open innovation not only stresses that knowledge is both plentiful and widely distributed across the globe (Chesbrough et al., 2006).

The literature on this topic acknowledges many problems associated with access and acquisition of knowledge outside the company such as identification of necessary knowledge sources, efficient scaling, and establishing technology markets. All these challenges create obstacles to the use of open innovation approach in companies. Chesbrough (2006) identify innovation intermediaries as organisations aimed at integration of different sources of knowledge and consult companies on how to use benefits of external, internal flows of knowledge. The Open Innovation concept acknowledges that in the innovation process the main actors are companies (supply-side stakeholders, for example, industry associations and knowledge-based businesses) and scientific institutions.

Meanwhile, there is a rise of a new participant in the innovation process - users playing a crucial role in open source communities or in shaping software products. Many studies 
have highlighted the significance of innovation intermediaries in the user-side activities. "Intermediate users, local experts and 'tailors' facilitate, configure and broker systems, usages and knowledge about systems and their deployments, helping users to domesticate them and suppliers to respond to actual, realised uses." (Stewart \& Hyysalo, 2008).

\section{Living Lab as new type of innovation intermediary}

A particular attention should be paid to a new form of innovation intermediaries - socalled Living Labs, which have spread across Sweden, Belgium, Finland and Spain since its creation in 2006.

Currently, throughout the world there are more than a hundred of Living Labs. This mediation service was created mainly as a public-private partnership to provide potential benefits to a region where user-driven innovations are being integrated into the collaborative process of creating new services, products and infrastructure. They represent a form of innovative cooperation, focused on the creation of functional areas where all stakeholders involved in public-private partnership (e.g. universities, government agencies, institutions and society) can interact for the purpose of creating, prototyping and testing of new technological products in real-time. This resulted in the emergence of a general platform to accelerate the innovation process and ensure the provision of medium- and long- term services for the development of new technologies that support innovation processes within various organizations.

Now we shall consider Living Labs more deeply as a type of the intermediaries acting as system builders of larger networks of organizations. Living Labs were formed mainly as Public-Private Partnerships to enforce regional advantages, where innovations are integrated within the collaboration process of creation of new services, products and infrastructures.

As innovation intermediaries Living Labs perform the following functions:

1. Facilitating cooperation in the field of research. Living Labs act as connectors, seeking technological complementarity and generating links on this basis. At the same time, they contribute to cooperation through medium- and long-term studies of possible types of technologies with all stakeholders, including future users, who are brought in at the research and development stage.

2. Providing complementary services to stakeholders. As a rule, research and development centers are continually expanding their range of services through the inclusion of additional activities, such as rationale for a project, inspection, marketing analysis and so on. The feature of Living labs is that they offer complementary services, including not only the creation and development of technologies, but also the provision of experimental platforms with a large number of users who are involved in the joint invention process by using prototypes of products.

3. Linking science and the state. As the intermediaries Living Labs contribute to the development of individual regions initiated by universities and public authorities sharing a desire to collaborate over science-driven innovations. Although few in numbers, these kinds of Living Labs aim to accelerate the development of new technologies in regions, promote certain directions of research and create synergistic effects between regional actors.

In general, the main functions performed by these innovation intermediaries are the following: development of knowledge, formation of new markets, development of the external economy and resource mobilization.

Living Labs represent a distinct type of intermediary configuration using their absorptive capacity to recognize, assimilate and apply external knowledge from users, universities, research centers, entrepreneurs and private organizations, to develop new innovations (Lopez-Vega and Vanhaverbeke, 2010). 
Apparently, Living Labs represent a prominent type of innovation intermediaries that can generate value and capture it by identifying new external knowledge, assimilate and apply it, as well as identify emerging technology demands in close collaboration with users. Living Labs require an intensive level of involvement from participating organizations. Living Labs are close to new science and technology, provided a high level of user participation. It invites universities, organizations, entrepreneurs and many users to take part in the innovation process requiring a great deal of resources in the course of the latter.

It is important that Living Labs help firms identify, articulate and codify external knowledge. This is an alternative explanation to principal frames such as alliances and partnerships and supplier relations. Firms could use innovation intermediaries to complement firm's open innovation activities in technology markets and remove managerial barriers such as searching and selecting external knowledge, information asymmetry and others.

These innovation intermediaries have many advantages for the established technology and idea markets as they are focused on value creation with using the Web-mediated model to involve a lot of problem solvers including contract laboratories, university faculties, research institutes, technology-based companies and other stakeholders.

Huston and Sakkab (2006) argue that these intermediaries reduce the costs and accelerate the speed of obtaining all solutions or new product concepts, create new company connections outside an original technological problem and a field of expertise, and contribute to the creation of knowledge from a broad range of solution providers.

Companies collaborate with Living Labs through its knowledge sourcing services like the search of new solutions from a network of external solution providers interested in collaborative partnership. It is worth noticing that this process may be hampered by managerial obstacles such as a lack of internal management leading the knowledge acquisition process. The knowledge acquisition process consists of six intermediation phases: 1) the need for identification; 2) the need for triangulation; 3) the need for specification; 4) search and collection; 5) evaluation; and 6) selection of solutions (LopezVega, 2013).

At the first phase, companies select innovation projects, decide to use innovation intermediaries for some reasons, involve other departments or business units, purchase everything necessary in the intermediation process and provide insights to reinforce the project's performance. There may be the following types of requests for proposal at this phase: new strategic initiatives, new product development, cost or quality improvement, market scanning for insights, technical gaps or implementation issues, fundamental scientific research.

The second phase is comprehending the external knowledge acquisition process and crafting the innovation problem.

The third phase includes description of the innovation problem's specifics and checking for confidentiality and anonymity. Intermediaries in this case help companies to 'focus' on the problem, explain its "technical" requirements to a broader audience, reveal companies' "relationships" expectations (academic researchers, entrepreneurs, labs), reveal their "commercial" needs, i.e. the ability to scale up, long-term supply, clarifying funding intentions for the external solution and IP expectations maintaining company's confidentiality for the selected project.

The fourth phase includes identifying solution providers, disseminating the challenge, giving feedback on received solutions, compelling and summarizing solutions for clients.

The next phase is initial internal evaluation of responses, having non-confidential conversations between parties and negotiating solutions.

The last phase is devoted to making decisions on integrating some external solutions according to the following conditions: a mature technological solution, a mid-stage 
technological solution, a novel solution, the ability to scale up, i.e. logistic, manufacturing, a solution that matches your budget, experience in proposed technologies, i.e. credibility, resources, financial stability (Vasin \& Gamidullaeva, 2017c).

Of particular interest, from our point of view, is a combination of the innovation ecosystem concept and the institution of innovation intermediation within an innovation ecosystem of a region. In the traditional sense, innovation intermediaries are understood as intermediaries acting at the company level between knowledge producers and consumers of knowledge (companies that want to solve specific problems) in order to facilitate conclusion of bilateral agreements between them. Currently, the concept of innovation intermediaries has undergone significant changes, and includes all those members who operate at the level of clusters, networks, businesses, societal actors, dealing with complex problems and even the innovation system as a whole. It has thus become reasonable and conceivable to implement the new approach to innovation intermediation, namely systemic innovation intermediation, whose members are working to create opportunities for innovation at a higher level of the innovation system, for example, at the level of subjects of the Russian Federation or at the country level.

These systemic innovation intermediaries perform a more independent role, working to facilitate innovation at a higher system-level, such as a specific sector, region or nation. According to Klerkx (2012) the term "systemic innovation intermediary" may be defined as an entity that operates at the interface between multiple innovation actors, working to facilitate and coordinate innovation activities at the system level.

Such mediators accelerate the innovation process, helping to cope with weaknesses of the innovation system, contributing to the implementation of innovations. They are able to overcome obstacles and difficulties encountered by companies such as underdeveloped markets, unregulated institutional incentives, poorly structured networks of economic actors and limited resources to support the innovation processes. Thus, system innovation intermediaries create coherence within the system and act as a coordinator in creating new opportunities and dynamism within the innovation system, acting as a catalyst for innovation processes. Of particular interest is the fact that they also perform a strategic role in coordinating and managing the innovation process, including coordination of the efforts form the industry, political authorities and research institutes.

Thus, the main objective of innovation intermediaries should be as follows: to find suitable solvers of problems that are the most appropriate for the economic agents involved in the search for solutions. In this case, the intermediaries should have adequate experience and have significant social capital. Ultimately, we need to move towards systemic intermediation, which will form a collaboration culture of the innovation society and will create a climate of trust among economic actors and promote confidence in institutions supporting the development of innovations. This may require platforms where actors, including the government, knowledge institutes, social organizations and companies come together to design new products. This association must be accompanied by an adequate process control and high quality strategic thinking (technological evaluation, benchmarking, monitoring studies, foresight). The main activities of such intermediaries include: foresight study, building networks for development, diffusion and implementation of innovations, development of tools and methods to identify, elaborate and implement joint innovation opportunities.

\section{Conclusion}


It is well known that companies try to implement a lot of strategies for external knowledge acquisition in order to improve and increase their competitiveness. In this regard, companies could collaborate with innovation intermediaries with the aim to search and acquire external knowledge. It is crucial that new types of intermediaries can provide firms with such services as knowledge articulation and codification. This is significantly important because companies could increase the scope of solutions and reduce the time to spot them in unknown technology markets.

Thus, innovation intermediaries should be considered as the basic control object within regional innovation ecosystems, providing complex support to all interested stakeholders, which is significantly important at all stages of the innovations' life span, including the subcontract stage of the innovation process.

The reported study was funded by RFBR according to the research project No. 18-01000204.

\section{References}

1. H. Chesbrough, Open Innovation: The new Imperative for Creating and Profiting from Technology (Harvard Business School Press, Boston, Massachussetts, 2003)

2. H. Chesbrough, California Management Review, 45, 3 (2003)

3. W. M. Cohen, D. A. Levinthal, Administrative Science Quarterly, 35, 1 (1990)

4. A. Folstad, eJov, 10 (SI on Living Labs) (2008)

5. P. A. Gloor, Collaborative Innovation through Swarm Creativity (Swarm Creativity 2006)

6. O. G. Golichenko, The main factors of development of the national innovation system (Nauka, Moscow, 2011)

7. J. Howells, Research Policy, 35, 5 (2006)

8. L. Huston, N. Sakkab, Harvard Business Review, 84, 3 (2006)

9. L. Klerkx, Improving Agricultural Knowledge and Innovation Systems (2012)

10. H. Lopez-Vega, Open innovation: Organizational practices and policy implications (Hasselt University, 2013)

11. H. Lopez-Vega, W. Vanhaverbeke, An Open Innovation perspective on the role of innovation intermediaries in technology and idea markets (Academy of Management Annual Meeting. Montreal, Canada, 2010)

12. S. Metcalfe, R. Ramlogan, The Quarterly Review of Economics and Finance, 48, 2 (2008)

13. R.J.W. Tijssen, Research Policy, 26, 7-8 (1998)

14. H. Van Lente, M. Hekkert, R. Smits, and B. van Waveren, International Journal of Innovation Management, 7, 3 (2003)

15. S. M. Vasin, L. A. Gamidullaeva, Theoretical and Applied Economics, 4 (2017)

doi: 10.25136 / 2409-8647.0.0.20644.

16. S. M. Vasin, L. A. Gamidullaeva, Innovations, 5, 223 (2017)

17. S. M. Vasin, L. A. Gamidullaeva, About Russian Regional Users' Innovation Based on Digital Information. In Pedro Isaias and Luisa Carvalho (Eds). User Innovation and the Entrepreneurship Phenomenon in the Digital Economy (IGI Global, Hershey, PA, 2017) doi: 10.4018/978-1-5225-2826-5 
18. P. Kivimaa, M. Martiskainen, Energy Efficiency, 11, 1 (2017). https://doi.org/10.1007/s12053-017-9547-y 